\title{
EDUCATION
}

\section{Key skills for newly qualified dentists: an evaluation of a West Midlands initiative}

\author{
V. R. Firmstone 1, A. D. Bullock ${ }^{2}$, J. Bedward ${ }^{3}$, J. W. Frame ${ }^{4}$, J. Hall ${ }^{5}$
}

\section{'The West Midlands Key Skills model provides a framework for learning during the VT year.'}

Introduction: This paper reports an evaluation of the West Midlands Key Skills initiative that provides a framework for learning during vocational training (VT).

Method: The 48 vocational dental practitioners (VDPs) who began their VT in August 2001 in the West Midlands were surveyed at the start and end of training ( 45 completed both surveys). They rated their confidence and experience in the 31 components of the Six Key Skills on visual analogue scales. Views were elicited in a survey of both VDPs (47 returns) and their general practice trainers (44 returns). Semi-structured interviews were also held with a stratified sample of 9 trainers and all four VT advisors.

Results: In terms of VDP progress, a statistically significant increase in confidence and experience was found in each of the 31 components. Six themes were identified in the views data. (i) Supporting the development of Key Skills; (ii) workload implications; (iii) the 'right' six? (iv) links with the advanced diploma (MFGDP(UK)); (v) assessment of VT; and, (vi) consistency and quality assurance.

Conclusion: VT successfully develops the confidence and experience of newly qualified dentists in the Six Key Skills and has been well received by the majority of VDPs, trainers and advisors in the West Midlands.

Almost all graduates of dentistry in UK spend the first year as vocational dental practitioners (VDPs), which is mandatory for those planning to work as principals within the General Dental Services of the NHS. The purpose of VT is to set the ethos for a lifetime of continuing professional development and create reflective practitioners who are aware of their own strengths and weaknesses. It is a year spent with an experienced practitioner (trainer) in an approved training practice. The educational input arises from weekly tutorials provided by the trainer and from 30 study days organised by the VT scheme advisors. There is no formal assessment of this period of training in England.

In 2001 the Committee on Vocational Training for England and Wales carried out its first review of $V^{1}{ }^{1}$. Part of this review included an inquiry carried out by Playdon which found that VT enabled the transition from dental student to dental practitioner within a supportive environment that was beneficial to both patients and VDPs. Recommendations included improvements in the curriculum for VT; improvements in trainer selection and training; increased monitoring of study days and the introduction of "a formal final assessment of the VDP". The need for assessment was also one of the recommendations of an earlier study undertaken by Frame et $a l^{2}$.

In response to their concern about "lack of regulation and the inability of trainers to deal with trainees lacking competence", Scotland has recently undertaken a three-year project to design a system of assessment for Dental Vocational Training/General Professional Training ${ }^{3,4}$. The emphasis has been on developing a competency-based system. The model includes four domains: clinical, communication, professionalism and managerial; 21 major competencies; and 147 supporting competencies. In essence, the document represents a curriculum which has been used to inform an assessment process.

The model developed in the West Midlands is 
known as The Six Key Skills and was first introduced in August 2000. Box 1 sets out these Key Skills and their 31 components. These provide a curriculum framework for learning during this period of training.

\section{Box 1: The Six Key Skills}

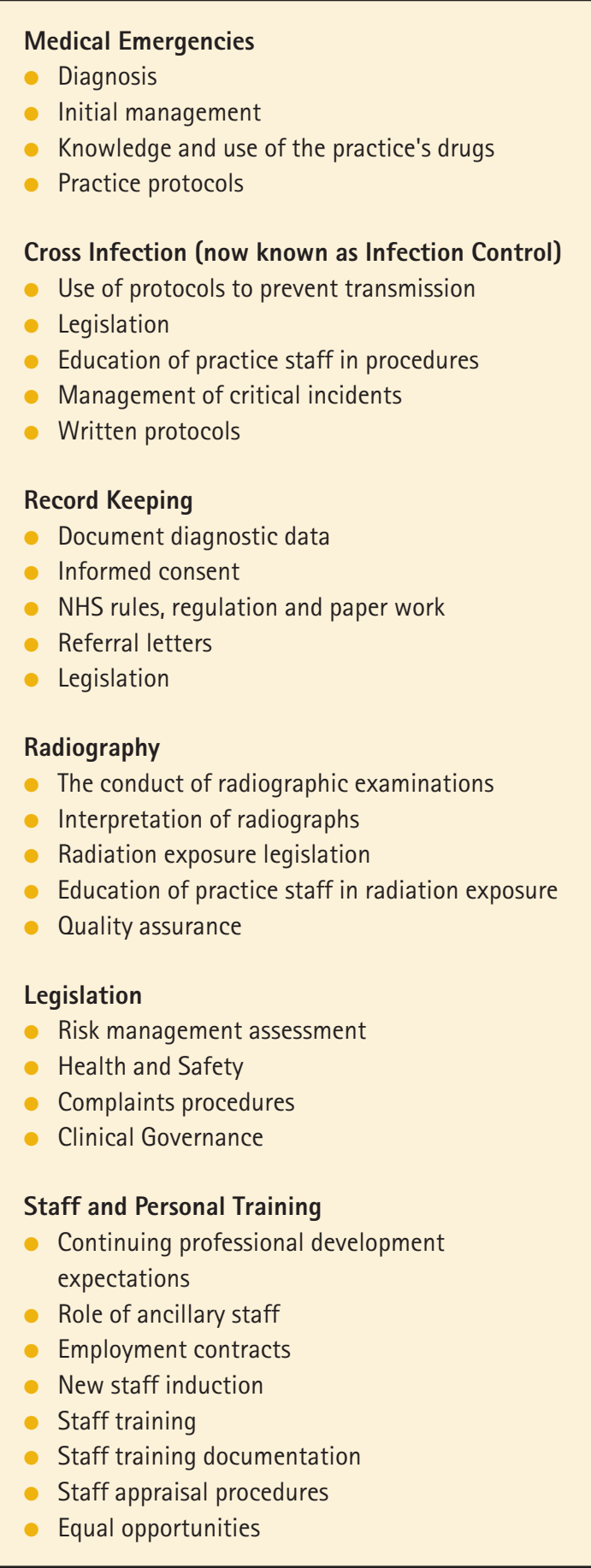

At the end of VT, trainees present an evidencedbased Key Skills portfolio which shows how these six areas are managed in their training practice. Coverage of the Six Key Skills and completion of the evidence-based portfolio is supported by the study day programme and by the trainers through the tutorial process. The Key Skills portfolio together with the clinical case report and the audit project form the coursework for the VT year. VDPs in the West Midlands who complete all parts of their coursework can optionally submit these three elements for assessment of the coursework module of the MFGDP(UK) at the end of the VT year.

The aim of the study reported here was to evaluate the implementation of the Key Skills initiative within VT in the West Midlands, evaluating VDPs' progress in the Key Skills and exploring the views of trainees, trainers and advisors ${ }^{5}$.

\section{'The aim of the study was to evaluate the \\ METHODS} implementation of the Key Skills initiative within VT in the West Midlands, evaluating VDPs' progress in the Key Skills and exploring the views of trainees, trainers and advisors'

The 48 vocational dental practitioners (VDPs) who started their VT in August 2001 in the West Midlands were surveyed at the start and end of training (45 completed both surveys). They rated their confidence and experience in each of the 31 components of the Six Key Skills on $100 \mathrm{~mm}$ visual analogue scales. Views were elicited in a survey of both VDPs (47 returns; 98\% response rate) and their general practice trainers (44 returns; 92\% response rate). These included a number of statements about Key Skills and vocational training, with a 4-point responseoption rating scale, ranging from strongly agree to strongly disagree. Semi-structured interviews were also held with a stratified sample of eight trainers and all four VT advisors.

Quantitative data were analysed using SPSS and Wilcoxon signed ranked test was employed to explore VDPs' change in confidence and experience. A standard approach to the analysis of qualitative interview data was adopted 6 . The interview transcripts were scrutinised to identify main issues, manually noting emerging themes and highlighting relevant text. The findings across transcripts were then compared and common themes identified.

\section{RESULTS}

\section{Progress in Key Skills}

At the end of VT, VDPs' self reported confidence median scores were $>=75$ in all 31 components of the Six Key Skills. For experience, scores were $>=75$ for 15 of the 31 components. Overall, components rated with lower levels of experience were mainly in medical emergencies and staff and personal training. The results are displayed in Table 1.

Looking across the 31 components, all the increases in both confidence and experience from baseline (start) to month 11 (end) were statistically significant (Wilcoxon).

Looking at the results by Key Skill, a number of observations may be made. In medical emergencies, respondents were much more confident than they were experienced, as is to be hoped.

Compared with other items for cross infection, at baseline experience was relatively low in educating staff in cross infection, managing critical incidents and knowledge of written protocols (confidence was low at baseline for this also). Despite significant increases in experience in managing critical incidents, experience in this area of cross infection remained relatively low at Month 11 (as might be hoped). 
Table 1: VDPs' confidence and experience median \# scores for each of the 31 components of the Six Key Skills at the start and end of VT.

\begin{tabular}{|c|c|c|c|c|}
\hline & \multicolumn{2}{|c|}{$\begin{array}{c}\text { Confidence } \\
\text { Median }\end{array}$} & \multicolumn{2}{|c|}{$\begin{array}{c}\text { Experience } \\
\text { Median }\end{array}$} \\
\hline & START & END & START & END \\
\hline Medical Emergencies & & & & \\
\hline Diagnosis & 54 & 75 & 13 & 48 \\
\hline Initial management & 57 & 78 & 10 & 49 \\
\hline Knowledge of drugs & 61 & 81 & 4 & 21 \\
\hline Practice protocols & 50 & 83 & 4 & 28 \\
\hline Cross Infection & & & & \\
\hline Prevention & 81 & 88 & 77 & 84 \\
\hline Legislation & 78 & 84 & 74 & 80 \\
\hline Educate staff & 65 & 86 & 9 & 77 \\
\hline Manage critical incidents & 73 & 85 & 15 & 40 \\
\hline Written protocols & 45 & 85 & 21 & 80 \\
\hline Record Keeping & & & & \\
\hline Document diagnostic data & 81 & 89 & 73 & 86 \\
\hline Informed consent & 69 & 85 & 30 & 77 \\
\hline NHS rules and regulations & 21 & 84 & 13 & 73 \\
\hline Write referral letters & 59 & 91 & 15 & 89 \\
\hline Legislation & 42 & 85 & 17 & 81 \\
\hline Radiography & & & & \\
\hline Radiographic examinations & 72 & 90 & 66 & 87 \\
\hline Interpret radiographs & 70 & 88 & 64 & 85 \\
\hline Legislation & 50 & 85 & 40 & 80 \\
\hline Educate staff about exposure & 56 & 85 & 12 & 67 \\
\hline Quality assurance & 35 & 82 & 7 & 73 \\
\hline Legislation & & & & \\
\hline Risk assessment & 27 & 87 & 8 & 80 \\
\hline Health and safety legislation & 28 & 83 & 9 & 76 \\
\hline Complaints procedures & 28 & 85 & 5 & 43 \\
\hline Clinical governance & 32 & 85 & 7 & 72 \\
\hline Staff and Personal Training & & & & \\
\hline $\mathrm{CPD}$ requirements & 72 & 87 & 11 & 80 \\
\hline Role of ancillary staff & 72 & 85 & 38 & 81 \\
\hline Employment contracts & 43 & 75 & 8 & 41 \\
\hline New Staff Induction & 17 & 78 & 7 & 51 \\
\hline Staff training & 38 & 78 & 5 & 61 \\
\hline Staff training documentation & 16 & 78 & 6 & 50 \\
\hline Staff appraisal & 19 & 77 & 5 & 36 \\
\hline Equal opportunities & 72 & 85 & 15 & 44 \\
\hline
\end{tabular}


Table 2: VDPs' views on support for developing key skills.

\begin{tabular}{|l|c|}
\hline Statement & $\begin{array}{c}\text { \% strongly/ } \\
\text { agreeing } \\
\text { (n=48) }\end{array}$ \\
\hline My trainer has given me good support in developing these Key Skills & 81 \\
The advisor has given me good support in learning about these Key Skills & 61 \\
My trainer has given me good support in putting together my portfolio & 58 \\
I am concerned about differences in how trainers support the development of Key Skills & 46 \\
\hline
\end{tabular}

\section{'Trainers were} initially apprehensive about increased

workload as a result of this initiative but it transpired that the main input for the VDPs came from the study days. Almost all of these trainers (96\%) agreed that "Key Skills are a good idea".'
(Source: VDPsurvey)

The record keeping results show that at baseline, experience was relatively low in informed consent, ability to write referral letters, understanding rules and regulations and knowledge of legislation for record keeping (confidence was low for the latter two at baseline also). Confidence and experience was high in all areas of record keeping at Month 11.

Compared with other items in radiography, knowledge of legislation related to radiation exposure, educating staff in the dangers of exposure, and implementing quality assurance programmes were relatively low for confidence and experience at baseline. Median scores for all items were relatively high at Month 11.

Confidence and experience was low for all areas of legislation at baseline. Although the increase was statistically significant, experience of understanding complaint procedures remained relatively low at Month 11.

Experience of all aspects of staff and personal training were relatively low at baseline, and confidence was particularly low for most of them except awareness of CPD requirements, understanding role of ancillary staff and understanding equal opportunities. At Month 11, confidence was high for all aspects and experience was high for CPD requirements and the role of ancillary staff and reasonably high for the ability to train staff. However, experience in the majority of staff and personal training aspects remained relatively low.

It is striking that the increases in both confidence and experience between the start and end of VT were statistically significant for all 31 elements of the Six Key Skills. The findings show how important VT was in developing experience. The self-ratings of experience in many items of the Key Skills were very low at the start of VT. VT successfully developed the confidence and experience of these newly qualified dentists in each of the Six Key Skills.

\section{Views of Key Skills}

Supporting the development of Key Skills

In practice, the advisors provided the main input on Key Skills through the study day programme. In an open question on the VDP survey VDPs described study days as "useful", "helpful", "essential" and as providing "a great learning opportunity".

Trainers were free to adopt their own approach to supporting Key Skills. A typical approach was to focus on two Key Skills per term. Both VDPs and their trainers felt that, of the Key Skills, 'legislation' required the highest level of support. Least support was thought to be needed for medical emergencies.

VDPs were satisfied with the level of support provided by advisors and trainers, but many (46\%) admitted to not being clear about what should go in the portfolio and were concerned about difference in trainer support (Table 2).

\section{Workload implications}

Trainers were initially apprehensive about increased workload as a result of this initiative but it transpired that the main input for the VDPs came from the study days. Almost all of these trainers (96\%) agreed that "Key Skills are a good idea".

In the VDP questionnaire, 88\% thought that Key Skills had increased their workload. The bulk of this additional work was associated with the compilation of the portfolio, which had to be completed mainly in the VDP's own time. However, most VDPs (66\%) did not consider that the VT year had been harder then expected and 69\% agreed that "Key Skills are a good idea”.

For advisors ensuring that Key Skills were covered at study days was not viewed as extra work:

'I don't see it as extra work, I see it as being a framework in which to organise my study days around. ... If you don't know about cross infection control, radiography and legislation and how to manage medical emergencies, I haven't 
been doing my job right. ... So the fact that somebody's called it Six Key Skills doesn't worry me; it's just something I would have to teach them anyway' (Advisor).

Most of the extra work for advisors involved checking VDPs' Key Skills portfolios and providing feedback.

\section{The right six?}

From the interview data, all the advisors considered that all six Key Skills were important areas and some suggested 'communication skills' could be an addition. Most of the trainers interviewed recognised the importance of the six particularly because of their relationship with clinical governance. However, different views were held on which Key Skills should be prioritised and other skills were identified including: clinical skills, financial management, judgement and diagnosis, treatment plans and, patient management.

\section{Links with the advanced diploma (MFGDP(UK))}

Seventy-seven percent of trainers and 73\% of VDPs reported to welcome the opportunity to submit the Key Skills portfolios to gain exemptions for MFGDP(UK) coursework. However, four trainers expressed concerns, for example that it encourages dentists to take the MFGDP(UK) too early in their career and detracts from a focus on clinical skills.

\section{Assessment of VT}

Advisors, trainers and most VDPs saw Key Skills as a step towards the assessment of VT. Most advisors viewed the introduction of assessment into VT positively. More mixed views were held by trainers. Just $46 \%$ of trainer questionnaire respondents strongly/agreed with the statement: 'I would welcome the formal assessment of VT.' However, in interview, the majority $(66 \%)$ of trainers indicated a positive view to the assessment of VT although some of these thought that assessment should be based on competency in clinical skills not on the Key Skills portfolio.

\section{Consistency and quality assurance}

From the trainers' questionnaire it was clear that most held positive views about the contribution of Key Skills to improvement in the structure and consistency of training within VT. Specifically, the vast majority of the trainers surveyed (93\%) felt that Key Skills improved the structure of training for VDPs. Ninety-one percent reported that Key Skills supported their role as trainer and 79\% thought that Key Skills promotes greater consistency between training practices. In interviews, advisors expressed similar views and considered that Key Skills provided increased structure to study days.

However, VDPs were concerned over differences between trainers in the level of support they provide in Key Skills (Table 2).

\section{DISCUSSION}

Statistically significant increases in levels of confidence and experience among VDPs in all elements of

\section{'Although most VDPs recorded a high level of satisfaction with the amount of support received from their trainers, there was concern about differences between trainers."}

the Six Key Skills occurred, clearly demonstrating that the VT year develops these skills in the recent dental graduate.

The findings show that the Key Skills programme gained the support of the trainers. This is particularly welcome given their starting point: most trainers were initially unenthusiastic about Key Skills. In part, this was attributed to their fears of an increased workload. The experience of training with Key Skills proved less difficult than anticipated.

The importance of these key skills was recognized although there were also suggestions for additions. Some of these (including diagnosis and technical skills) should be demonstrated in the other elements of the coursework (the clinical case and the audit project). If the Key Skills framework is subject to periodic review then whether the framework should be expanded to specifically include communication skills (as in the Scottish model ${ }^{3,4}$ ) could be considered.

Although most VDPs recorded a high level of satisfaction with the amount of support received from their trainers, there was concern about differences between trainers. This highlights the importance of study days in providing a structured, comprehensive approach to Key Skills.

One suggestion for strengthening the quality of the support offered by trainers was to clarify the requirements of the evidence-based Key Skills portfolio. Trainers might be encouraged to produce evidence-based Key Skills portfolios on their own practices. This would give them a good understanding of the nature of the task. It would also provide evidence that the training practice was fulfilling the requirements of clinical governance.

It would also be beneficial for the training of trainers to be strengthened. Although the study days provide an effective framework for the Key Skills Initiative, the trainer as a supportive mentor is instrumental to using the portfolio as an effective learning tool ${ }^{7}$. The importance of trainer training is obviously raised if assessment of the VDP becomes a requirement for the successful completion of VT.

The issue of assessment in VT is contentious. The sample of trainers in this study held mixed views. About half of those surveyed would not welcome formal assessment of VT. For some this was because their VDP was qualified to practice upon graduation and so they felt assessment of VT lacked credibility. Clearly though, assessment of VT is being considered at a national level ${ }^{1}$. We advocate the integration of the Key Skills portfolio within part of a final assessment within VT. Feedback on the Key Skills portfolio might need to be undertaken at key points in the year, to ensure satisfactory progress and safeguard against plagiarism. This would conform to the method identified by Driessen et al7 which involved 


\section{Box 2: Integrating the Key Skills portfolio into an assessment of VT}

1. VDP drafts parts of the portfolio and discusses with the trainer who provides formative feedback over the course of the year.

2. VDP submits a first full draft to the advisor for more formative feedback. Again, the advisor reviews the portfolio in light of the MFGDP(UK) criteria.

3. VDP redrafts the portfolio following the advisor's feedback and the final portfolio is submitted to their advisor at the end of $\mathrm{VT}$.

4. Successful completion (or assessment) of the portfolio using the standard designed by the MFGDP(UK) becomes part of the ratification process for 'satisfactory' completion of VT.

5. Those unsuccessful are able to resubmit within a specified period and could be supported in this by joining a study group. Given that completion of VT is an essential pre-requisite for obtaining a GDS number, VDPs would be required to work as assistants in the meantime.

6. The VDP can choose to submit their portfolios for part exemption of the MFGDP(UK).

'Within the context of gathering momentum for increased assessment, the Key Skills development can be viewed as a timely, proactive response.' 'multiple portfolio discussion points' (p. 20) so that end of year assessment does not lead to unexpected outcomes.

The Key Skills portfolio, along with the two other components of coursework (clinical case and audit), are graded by MFGDP(UK) examiners. It would be feasible to set the minimum standard required in VT at this level.

The process could thus entail the steps outlined in Box 2.

We recognise that the introduction of a national standard for the successful completion of the portfolio is currently problematic, given that the coursework (portfolio, clinical audit and case) are not compulsory elements of VT. Steps 4 and 5 (Box II) require change at a national level. We note, however, that CVT has recently merged with the statutory Dental Vocational Training Authority for England and Wales (DVTA). Changes such as this, at a national level, and the developing role of the Primary Care Trusts (PCTs) may provide further impetus for discussion of these issues.

\section{CONCLUSION}

The findings demonstrate the success of the Key Skills initiative in gaining general acceptance from VDPs, trainers and advisors. The study also demonstrates the effectiveness of the programme in increasing levels of experience and confidence during VT. Generally, most respondents consider that the Key Skills initiative was introduced in response to increased demands for formal assessment within VT. Within the context of gathering momentum for increased assessment, the Key Skills development can be viewed as a timely, proactive response.
The project team is grateful for the financial support of the West Midlands Postgraduate Deanery for funding this project. The views and opinions expressed are the authors' and do not necessarily reflect those of the funders. We would also like to extend our thanks to those VDPs, trainers and advisors who gave their time to completing questionnaires and/or being interviewed. Particular acknowledgement is given to the advisors' who distributed and collected questionnaires from the VDPs during the study days.

1 Committee on Vocational Training for England and Wales. Review of Vocational Training in Dentistry for the Chief Dental Officers England and Wales. CVT, London 2002.

2 Frame JW, Bullock A D, Butterfield, S, Morris ZS and Belfield CR. An Evaluation of Assessment in Post-registration Dental Education (Final Report), Birmingham: The University of Birmingham. 1999.

3 NHS Scotland. Competencies for Dental Vocational Training/General Professional Training in Scotland. NHS Scotland 2002.

4 Prescott LE, McKinlay P, and Rennie J S. The development of an assessment system for dental vocational training and general professional training: A Scottish approach. BrDent J 2001; 190: 41- 44.

5 Firmstone V, Bedward J, Bullock A, Hall J and Frame J. Key Skills in Vocational Training: a West Midlands evaluation. Final Report. Birmingham: The University of Birmingham. 2003.

6 Coffey A and Atkinson P. Making Sense of Qualitative Data. Sage Publications, London. 1996.

7 Driessen EW, Van Tartwijk J, Vermunt JD and Van der Vleuten CPM. Use of portfolios in early undergraduate medical training. Medical Teacher 2003; 25: 18-23. 